\title{
A new pricing measure in the Barndorff-Nielsen \& Shephard model for commodity markets
}

\author{
Salvador Ortiz-Latorre \\ Department of Mathematics \\ University of Oslo \\ P.O. Box 1053, Blindern \\ Oslo, N-0316, Norway \\ salvadoo@math.uio.no \\ This talk was based on the research paper [4], which is a joint work with Fred Espen Benth. We are grateful \\ for the financial support from the project "Energy Markets: Modeling, Optimization and Simulation (EMMOS)", \\ funded by the Norwegian Research Council under grant Evita/205328.
}

\begin{abstract}
For a commodity spot price dynamics given by an Ornstein-Uhlenbeck process with Barndorff-Nielsen \& Shephard stochastic volatility, we price forward contracts using a new class of pricing measures, extending the classical Esscher transform, that simultaneously allow for change of level and speed in the mean reversion of both the price and the volatility.
\end{abstract}

\section{Introduction}

Benth and Ortiz-Latorre [3] analysed a structure preserving class of pricing measures for Ornstein-Uhlenbeck (OU) processes with applications to forward pricing in electricity markets. In particular, they considered multi-factor OU models driven by Lévy processes having positive jumps (so-called subordinators) or Brownian motions for the spot price dynamics and analysed the risk premium when the level and speed of mean reversion in these factor processes were changed.

In this work we present the results obtained in [4], where we continue this study for OU processes driven by Brownian motion, but with a stochastic volatility perturbing the driving noise. The stochastic volatility process is modelled again as an OU process, but driven by a subordinator. This class of stochastic volatility models were first introduced by Barndorff-Nielsen and Shephard [1] for equity prices, and later analysed by Benth [2] in commodity markets. The class of pricing measures we study here allows for a simultaneous change of speed and level of mean reversion for both the (logarithmic) spot price and the stochastic volatility process. The mean reversion level can be flexibly shifted up or down, while the speed of mean reversion can be slowed down. It significantly extends the classical Esscher transform, see Gerber and Shiu [8], which only allows for changes in the level of mean reversion.

The affine structure of the model can be exploited to reduce the forward pricing to solving a system of Riccati equations by resorting to the theory of Kallsen and MuhleKarbe [11]. The forward price becomes a function of both the spot and the volatility, and has a deterministic asymptotic dynamics when we are far from maturity. By careful analysis of the associated system of Riccati equations, we can study the implied risk premium of our class of measure change as a function of its parameters. The risk premium is defined as the difference between the forward price and the predicted spot price at 
maturity, and is a notion of great importance in commodity markets since it measures the price for entering a forward hedge position in the commodity (see e.g. Geman [7] for more on this). In particular, under rather mild assumptions on the parameters, we can show that the risk premium may change sign stochastically, and may be positive for short times to maturity and negative when maturity is farther out in time. This is a profile of the risk premium that one may expect in energy markets based on both economical and empirical findings, which cannot be obtained by using the Esscher transform.

\section{Mathematical model}

Suppose that $\left(\Omega, \mathcal{F},\left\{\mathcal{F}_{t}\right\}_{t \in[0, T]}, P\right)$ is a filtered probability space satisfying the usual hyphotesis, where $T>0$ is a fixed finite time horizon. On this probability space there are defined $W$, a standard Wiener process, and $L$, a pure jump Lévy subordinator with finite expectation, that is, a finite variation Lévy process with jumps supported on the positive axis and $\mathbb{E}[L(1)]<\infty$, or equivalently, a Lévy process with the following LévyItô representation $L(t)=\int_{0}^{t} \int_{0}^{\infty} z N^{L}(d s, d z), t \in[0, T]$, where $N^{L}(d s, d z)$ is a Poisson random measure with Lévy measure $\ell$ satisfying $\int_{0}^{\infty} z \ell(d z)<\infty$. We shall suppose that $W$ and $L$ are independent of each other. As we are going to consider an Esscher change of measure and geometric spot price models, we introduce an assumption on the existence of exponential moments of $L$.

Assumption 1 Suppose that

$$
\Theta_{L} \triangleq \sup \left\{\theta \in \mathbb{R}_{+}: \mathbb{E}_{P}\left[e^{\theta L(1)}\right]<\infty\right\}
$$

is a constant strictly greater than zero, which may be $\infty$.

In $\left(-\infty, \Theta_{L}\right)$ the cumulant (or log moment generating) function $\kappa_{L}(\theta) \triangleq \log \mathbb{E}_{P}\left[e^{\theta L(1)}\right]$ is well defined and analytic. As $0 \in\left(-\infty, \Theta_{L}\right), L$ has moments of all orders. Also, $\kappa_{L}(\theta)$ is convex, which yields that $\kappa_{L}^{\prime \prime}(\theta) \geq 0$ and, hence, that $\kappa_{L}^{\prime}(\theta)$ is non decreasing. Finally, as a consequence of $L \geq 0$,a.s., we have that $\kappa_{L}^{\prime}(\theta)$ is non negative. Moreover, we have that

$$
\kappa_{L}(\theta)=\int_{0}^{\infty}\left(e^{\theta z}-1\right) \ell(d z)<\infty, \quad \kappa_{L}^{(n)}(\theta)=\int_{0}^{\infty} z^{n} e^{\theta z} \ell(d z)<\infty, \quad n \in \mathbb{N},
$$

where $\kappa_{L}^{(n)}(\theta)$ denotes the $n$-th derivative of $\kappa_{L}(\theta)$.

Consider the OU-type processes

$$
\begin{aligned}
& X(t)=X(0)-\alpha \int_{0}^{t} X(s) d s+\int_{0}^{t} \sigma(s) d W(s), \\
& \sigma^{2}(t)=\sigma^{2}(0)+\int_{0}^{t}\left(\kappa_{L}^{\prime}(0)-\rho \sigma^{2}(s)\right) d s+\int_{0}^{t} \int_{0}^{\infty} z \tilde{N}^{L}(d s, d z),
\end{aligned}
$$

where $t \in[0, T], \alpha, \rho>0, X(0) \in \mathbb{R}, \sigma^{2}(0)>0$ and $\tilde{N}^{L}(d s, d z) \triangleq N^{L}(d s, d z)-d s \ell(d z)$ is the compensated version of $N^{L}(d s, d z)$.

\section{The change of measure}

We will consider a parametrized family of measure changes that will allow us to simultaneously modify the speed and the level of mean reversion in equations (2) and (3). The 
density processes of these measure changes are given by

$$
\left.\frac{d Q_{\bar{\theta}, \bar{\beta}}}{d P}\right|_{\mathcal{F}_{t}} \triangleq \mathcal{E}\left(\tilde{G}_{\theta_{1}, \beta_{1}}+\tilde{H}_{\theta_{2}, \beta_{2}}\right)(t), \quad t \in[0, T]
$$

where

$$
\begin{aligned}
& \tilde{G}_{\theta_{1}, \beta_{1}}(t) \triangleq \int_{0}^{t} \sigma^{-1}(s)\left(\theta_{1}+\alpha \beta_{1} X(s)\right) d W(s), \quad t \in[0, T] \\
& \tilde{H}_{\theta_{2}, \beta_{2}}(t) \triangleq \int_{0}^{t} \int_{0}^{\infty}\left(e^{\theta_{2} z}\left(1+\frac{\rho \beta_{2}}{\kappa_{L}^{\prime \prime}\left(\theta_{2}\right)} z \sigma^{2}(s-)\right)-1\right) \tilde{N}^{L}(d s, d z), \quad t \in[0, T],
\end{aligned}
$$

and $\mathcal{E}(\cdot)$ denotes the stochastic exponential, see Section 8, Chapter II in Protter [12]. The parameters $\bar{\beta} \triangleq\left(\beta_{1}, \beta_{2}\right)$ and $\bar{\theta} \triangleq\left(\theta_{1}, \theta_{2}\right)$ will take values on the following sets $\bar{\beta} \in[0,1]^{2}, \bar{\theta} \in \bar{D}_{L} \triangleq \mathbb{R} \times D_{L}$, where $D_{L} \triangleq\left(-\infty, \Theta_{L} / 2\right)$ and $\Theta_{L}$ is given by equation (1). Note that when $\beta_{1}=\beta_{2}=0$ we get the Esscher transform, see Benth and Sgarra $[6]$. The following theorem ensure that we have a bona fide change of probability measure.

Theorem 2 Let $\theta \in \bar{D}_{L}, \bar{\beta} \in[0,1]^{2}$. Then $\mathcal{E}\left(\tilde{G}_{\theta_{1}, \beta_{1}}+\tilde{H}_{\theta_{2}, \beta_{2}}\right)=\left\{\mathcal{E}\left(\tilde{G}_{\theta_{1}, \beta_{1}}+\tilde{H}_{\theta_{2}, \beta_{2}}\right)(t)\right\}_{t \in[0, T]}$ is a strictly positive true martingale under $P$.

Then, by Girsanov's theorem for semimartingales (Theorems 1 and 3, pages 702 and 703 in Shiryaev $[\mathbf{1 3}])$, under $Q_{\bar{\theta}, \bar{\beta}}$, the processes $X(t)$ and $\sigma^{2}(t)$ become

$$
\begin{aligned}
& X(t)=X(0)+\int_{0}^{t}\left(\theta_{1}-\alpha\left(1-\beta_{1}\right) X(s)\right) d s+\sigma(t) W_{Q_{\bar{\theta}, \bar{\beta}}}(t), \\
& \sigma^{2}(t)=\sigma^{2}(0)+\int_{0}^{t}\left(\kappa_{L}^{\prime}\left(\theta_{2}\right)-\rho\left(1-\beta_{2}\right) \sigma^{2}(s)\right) d s+\int_{0}^{t} \int_{0}^{\infty} z \tilde{N}_{Q_{\bar{\theta}, \bar{\beta}}^{L}}^{L}(d s, d z),
\end{aligned}
$$

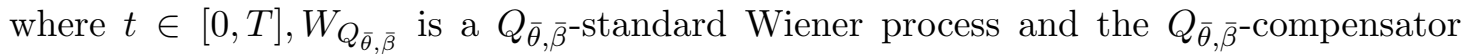
measure of $\sigma^{2}$ (and $L$ ) is

$$
v_{Q_{\bar{\theta}, \bar{\beta}}}^{\sigma^{2}}(d t, d z)=v_{Q_{\bar{\theta}, \bar{\beta}}}^{L}(d t, d z)=H_{\theta_{2}, \beta_{2}}(t, z) \ell(d z) d t .
$$

Remark 3 Under $Q_{\bar{\theta}, \bar{\beta}}, \sigma^{2}$ still satisfies the Langevin equation with different parameters, that is, the measure change preserves the structure of the equations for $\sigma^{2}$. However, the process $L$ is not a Lévy process under $Q_{\bar{\theta}, \bar{\beta}}$, but it remains an affine semimartingale, see $[\mathbf{1 0}]$. The equation for $X$ is the same under the new measure but with different parameters.

\section{Geometric spot model}

For modeling the commodity spot price $S$ we consider a geometric model, that is,

$$
S(t)=\Lambda_{g}(t) \exp (X(t)), \quad t \in\left[0, T^{*}\right],
$$

where $T^{*}>0$ is a fixed time horizon. The process $\Lambda_{g}$ is assumed to be deterministic and it accounts for the seasonalities observed in the spot prices. Using a risk-neutral pricing argument (see Benth, Šaltytė Benth and Koekebakker [5]), under the assumption of deterministic interest rates, the forward price at time $0 \leq t$, with time of delivery $T$ with $t \leq T<T^{*}$, is given by $F_{Q}(t, T) \triangleq \mathbb{E}_{Q}\left[S(T) \mid \mathcal{F}_{t}\right]$. The risk premium for forward prices with a fixed delivery time is defined by the following expression

$$
R_{Q}^{F}(t, T) \triangleq \mathbb{E}_{Q}\left[S(T) \mid \mathcal{F}_{t}\right]-\mathbb{E}_{P}\left[S(T) \mid \mathcal{F}_{t}\right]
$$


Here, $Q$ is any probability measure equivalent to the historical measure $P$ (not necessarily a martingale measure) and $\mathcal{F}_{t}$ is the market information up to time $t$. In what follows we will use the probability measure $Q=Q_{\bar{\theta}, \bar{\beta}}$ in order to compute the forward prices and the risk premium. The following is a sufficient condition to ensure that $S(T) \in L^{1}(P)$.

Assumption $4(\mathcal{P})$ We assume that $\alpha, \rho>0$ and $\Theta_{L}$ satisfy

$$
\frac{1}{2 \rho}\left(\frac{\rho}{2 \alpha}\right)^{\frac{1}{1-\frac{\rho}{2 \alpha}}} \leq \Theta_{L}-\delta
$$

for some $\delta>0$.

Based on an affine tranform formula for affine semimartingales, see Kallsen and Muhle-Karbe [11], we can provide semi-explicit expressions for $\mathbb{E}_{Q}\left[S(T) \mid \mathcal{F}_{t}\right]$.

Theorem 5 Let $\bar{\beta}=\left(\beta_{1}, \beta_{2}\right) \in[0,1]^{2}, \bar{\theta}=\left(\theta_{1}, \theta_{2}\right) \in \bar{D}_{L}$ and $T>0$. Assume that there exist functions $\Psi_{i}^{\bar{\theta}, \bar{\beta}}, i=0,1,2$, belonging to $C^{1}([0, T] ; \mathbb{R})$, satisfying the generalised Riccati differential equation

$$
\begin{aligned}
& \frac{d}{d t} \Psi_{0}^{\bar{\theta}, \bar{\beta}}(t)=\theta_{1} \Psi_{2}^{\bar{\theta}, \bar{\beta}}(t)+\kappa_{L}\left(\Psi_{1}^{\bar{\theta}, \bar{\beta}}(t)+\theta_{2}\right)-\kappa_{L}\left(\theta_{2}\right), \\
& \frac{d}{d t} \Psi_{1}^{\bar{\theta}, \bar{\beta}}(t)=-\rho \Psi_{1}^{\bar{\theta}, \bar{\beta}}(t)+\frac{\left(\Psi_{2}^{\bar{\theta}, \bar{\beta}}(t)\right)^{2}}{2}+\frac{\rho \beta_{2}}{\kappa_{L}^{\prime \prime}\left(\theta_{2}\right)}\left(\kappa_{L}^{\prime}\left(\Psi_{1}^{\bar{\theta}, \bar{\beta}}(t)+\theta_{2}\right)-\kappa_{L}^{\prime}\left(\theta_{2}\right)\right), \\
& \frac{d}{d t} \Psi_{2}^{\bar{\theta}, \bar{\beta}}(t)=-\alpha\left(1-\beta_{1}\right) \Psi_{2}^{\bar{\theta}, \bar{\beta}}(t),
\end{aligned}
$$

with initial conditions $\Psi_{0}^{\bar{\theta}, \bar{\beta}}(0)=\Psi_{1}^{\bar{\theta}, \bar{\beta}}(0)=0, \Psi_{2}^{\bar{\theta}, \bar{\beta}}(0)=1$ and the integrability condition $\sup _{t \in[0, T]} \kappa_{L}^{\prime \prime}\left(\theta_{2}+\Psi_{1}^{\bar{\theta}, \bar{\beta}}(t)\right)<\infty$. Then,

$$
\mathbb{E}_{Q}\left[\exp (X(T)) \mid \mathcal{F}_{t}\right]=\exp \left(\Psi_{0}^{\bar{\theta}, \bar{\beta}}(T-t)+\Psi_{1}^{\bar{\theta}, \bar{\beta}}(T-t) \sigma^{2}(t)+\Psi_{2}^{\bar{\theta}, \bar{\beta}}(T-t) X(t)\right),
$$

and

$$
\begin{aligned}
R_{Q}^{F}(t, T)=\mathbb{E}_{P}\left[S(T) \mid \mathcal{F}_{t}\right] & \left\{\operatorname { e x p } \left(\Psi_{0}^{\bar{\theta}, \bar{\beta}}(T-t)-\int_{0}^{T-t} \kappa_{L}\left(e^{-\rho s} \frac{1-e^{-(2 \alpha-\rho) s}}{2(2 \alpha-\rho)}\right) d s\right.\right. \\
+ & \left(\Psi_{1}^{\bar{\theta}, \bar{\beta}}(T-t)-e^{-\rho(T-t)} \frac{1-e^{-(2 \alpha-\rho)(T-t)}}{2(2 \alpha-\rho)}\right) \sigma^{2}(t) \\
& \left.\left.+\left(\Psi_{2}^{\bar{\theta}, \bar{\beta}}(T-t)-e^{-\alpha(T-t)}\right) X(t)\right)-1\right\},
\end{aligned}
$$

for $t \in[0, T]$.

The applicability of Theorem 5 is quite limited as it is stated. This is due to the fact that it is very difficult to see a priori if there exist functions $\Psi_{i}^{\bar{\theta}, \bar{\beta}}, i=0,1,2$ belonging to $C^{1}\left([0, T] ; \mathbb{R}^{2}\right)$ satisfying equation $(6)$. One has to study existence and uniqueness of solutions of equation (6) and the possibility of extending the solution to arbitrary large $T>0$. The idea is to provide sufficient conditions on $(\bar{\theta}, \bar{\beta})$ ensuring that $\Psi_{i}^{\bar{\theta}}, \bar{\beta}(t), i=$ $0,1,2$ remain bounded and do not explode if finite time. We have the following result:

Theorem $6 \Lambda^{\theta, \beta, a}:\left[0, \Theta_{L}-\theta\right) \rightarrow \mathbb{R}$ be the function defined by

$$
\Lambda^{\theta, \beta, a}(u)=-\rho u+a+\frac{\rho \beta}{\kappa_{L}^{\prime \prime}(\theta)}\left(\kappa_{L}^{\prime}(u+\theta)-\kappa_{L}^{\prime}(\theta)\right),
$$

where $a \geq 0,(\theta, \beta) \in D_{L} \times(0,1)$ and consider the set

$$
\mathcal{D}_{b}(a)=\left\{(\theta, \beta) \in D_{L} \times(0,1): \exists u \in\left[0, \Theta_{L}-\theta\right) \quad \text { s.t. } \quad \Lambda^{\theta, \beta, a}(u) \leq 0\right\} .
$$


If $\left(\theta_{2}, \beta_{2}\right) \in \mathcal{D}_{b}(1 / 2)$ and $\left(\theta_{1}, \beta_{1}\right) \in \mathbb{R} \times[0,1)$ then $\left(\Psi_{0}^{\bar{\theta}, \bar{\beta}}(t), \Psi_{1}^{\bar{\theta}, \bar{\beta}}(t), \Psi_{2}^{\bar{\theta}, \bar{\beta}}(t)\right)$ are $C^{1}([0, T] ; \mathbb{R})$ for any $T>0$. Moreover,

$$
\begin{gathered}
\Psi_{0}^{\bar{\theta}, \bar{\beta}}(t) \longrightarrow \frac{\theta_{1}}{\alpha\left(1-\beta_{1}\right)}+\int_{0}^{\infty}\left\{\kappa_{L}\left(\Psi_{1}^{\bar{\theta}, \bar{\beta}}(t)+\theta_{2}\right)-\kappa_{L}\left(\theta_{2}\right)\right\} d s, \quad t \rightarrow \infty \\
\left(\Psi_{1}^{\bar{\theta}, \bar{\beta}}(t), \Psi_{2}^{\bar{\theta}, \bar{\beta}}(t)\right) \longrightarrow(0,0), \quad t \rightarrow \infty
\end{gathered}
$$

and

$$
t^{-1} \log \left\|\left(\Psi_{1}^{\bar{\theta}, \bar{\beta}}(t), \Psi_{2}^{\bar{\theta}, \bar{\beta}}(t)\right)\right\| \rightarrow \gamma, \quad t \rightarrow \infty
$$

where $\gamma=-\alpha\left(1-\beta_{1}\right)$ or $\gamma=-\rho\left(1-\beta_{2}\right)$.

An immediate consequence of the Theorem 6 is that the forward price will be equal to the seasonal function $\Lambda_{g}(T)$ in the long end, that is, when $\left(\theta_{2}, \beta_{2}\right) \in \mathcal{D}_{b}(1 / 2)$ and $\left(\theta_{1}, \beta_{1}\right) \in \mathbb{R} \times[0,1)$, it holds that

$$
\lim _{T \rightarrow \infty} \frac{F_{Q}(t, T)}{\Lambda_{g}(T)}=\exp \left(\frac{\theta_{1}}{\alpha\left(1-\beta_{1}\right)}+\int_{0}^{\infty}\left\{\kappa_{L}\left(\Psi_{1}^{\bar{\theta}, \bar{\beta}}(s)+\theta_{2}\right)-\kappa_{L}\left(\theta_{2}\right)\right\} d s\right) .
$$

In [4] we provide a comprehensive qualitatively analysis of the possible risk premium profiles that can be obtained using our change of measure. In particular, we show that is possible to generate risk profiles with positive values in the short end of the forward curve and negative values in the long end. Moreover, we show that the sign of the risk premium can change stochastically. These two features cannot be obtained by using the Esscher transform.

\section{References}

[1] Barndorff-Nielsen, O. E., and Shephard, N. (2001). Non-Gaussian Ornstein-Uhlenbeck models and some of their uses in economics. J. Roy. Stat. Soc. B, 63(2), pp. 167-241 (with discussion).

[2] Benth, F. E. (2011). The stochastic volatility model of Barndorff-Nielsen and Shepard in commodity markets. Math. Finance, 21, pp. 595-625.

[3] Benth, F. E. and Ortiz-Latorre, S. (2014). A pricing measure to explain the risk premium in power markets. SIAM J. Finan. Math., 5(1), pp. 685-728.

[4] Benth, F. E. and Ortiz-Latorre, S. (2015). A change of measure preserving the affine structure in the Barndoff-Nielsen and Shephard model for commodity markets. International Journal of Theoretical and Applied Finance, 18(6), 40 pages.

[5] Benth, F. E., Šaltytė Benth, J. and Koekebakker, S. (2008). Stochastic Modelling of Electricity and Related Markets. World Scientific, Singapore.

[6] Benth, F. E. and Sgarra, C. (2012). The risk premium and the Esscher transform in power markets. Stochastic Anal. Appl., 30 (1), pp. 20-43.

[7] Geman, H. (2005). Commodities and Commodity Derivatives, John Wiley \& Sons, Chichester.

[8] Gerber, H. U., and Shiu, E. S. W. (1994). Option pricing by Esscher transforms. Trans. Soc. Actuaries, 46, pp. 99-191 (with discussion).

[9] Kallsen, J. (2006). A didactic note on affine stochastic volatility models. In Yu. Kabanov, R. Liptser, and J. Stoyanov (eds.): From Stochastic Calculus to Mathematical Finance, pp. 343-368, Springer, Berlin.

[10] Kallsen, J. (2006). A didactic note on affine stochastic volatility models. In Yu. Kabanov, R. Liptser, and J. Stoyanov (eds.): From Stochastic Calculus to Mathematical Finance, pp. 343-368, Springer, Berlin.

[11] Kallsen, J. and Muhle-Karbe J. (2010). Exponentially affine martingales, affine measure changes and exponential moments of affine processes. Stoch. Proc. Applic., 120, pp. 163-181.

[12] Protter, P. E. (2004). Stochastic Integration and Differential Equations, 2nd Edition. Springer Verlag, Berlin Heidelberg New York.

[13] Shiryaev, A. N. (1999). Essentials of Stochastic Finance, World Scientific, Singapore. 